Published in final edited form as:

Am J Med Sci. 2015 January ; 349(1): 2. doi:10.1097/MAJ.0000000000000401.

\title{
Endothelial Dysfunction in Injury and Inflammation
}

\author{
Jim C. Oates, MD \\ Division of Rheumatology and Immunology, Department of Medicine, Medical University of South \\ Carolina, Charleston, South Carolina; and Rheumatology Section of the Medical Service and \\ Research Service of the Ralph H Johnson VA Medical Center, Charleston, South Carolina.
}

Welcome to the 2014 SSCI Presidential Symposium. Today's presentation will focus on the role of the endothelium in vascular and liver disease. Before I introduce the speakers, I would like to give some historical context. The vascular endothelial cell lining is the gateway between circulating blood and the extracellular space. It serves as an environmental sensor that becomes activated by signals and stimuli in both spaces. One of the first descriptions of endothelial cells as environmental sensors stemmed from the discovery of endothelium-derived relaxation factor, later realized to be nitric oxide (NO). Robert Furchgott was performing experiments designed to measure smooth muscle contraction in isolated arteries. Traditionally, endothelial cells and adventitia were removed from these preparations before experiments were performed. Arteries prepared in this standard fashion were known to contract in response to a stimulus of acetylcholine. However, by happenstance, when endothelial cells were not removed from the arteries before experiments, they would paradoxically relax. ${ }^{1}$ This demonstrated that endothelial cells translated messages from the vascular space into second signals to smooth muscle cells. We now know that $\mathrm{NO}$ is a critical mediator in this process.

What is becoming more apparent is the key role that the endothelium plays in vascular inflammation and damage. In response to infectious stimuli, chemotaxis, adhesion and transmigration of inflammatory cells into inflamed tissue are coordinated by the vascular endothelium and modulated by the product of endothelial nitric oxide synthase (eNOS). ${ }^{2}$ When the endothelium is activated and eNOS activity is reduced in the absence of infection, pathology often follows.

Our first speaker is Philip Shaul. Dr. Shaul received his Bachelor of Science from Dartmouth College and his Medical Degree from the University of Cincinnati. He completed his residency in Pediatrics at Children's Hospital Medical Center at the University of Cincinnati. He completed his fellowship in neonatal and perinatal medicine at Brown University. He was recruited to the University of Texas, Southwestern, where he rose through the ranks and now serves as the Director of the Center for Pulmonary and Vascular Biology and Professor in the Department of Pediatrics. His research focuses on the regulation of eNOS in vascular disease. His presentation ${ }^{3}$ focuses on the role of the endothelium in features of the metabolic

Correspondence: Jim C. Oates, MD, Division of Rheumatology and Immunology, Department of Medicine, Medical University of South Carolina, 96 Jonathan Lucas Street, Suite 816, MSC 637, Charleston, SC 29425 (oatesjc@ musc.edu).

The author has no financial or other conflicts of interest to disclose. 
syndrome, namely hypertension and insulin resistance. He reviews over a decade of work in his laboratory and others that demonstrates a critical role for C-reactive protein (CRP) signaling through $\mathrm{Fc}$ receptor gamma IIB to reduce the protective effect of eNOS NO production in endothelial cells. CRP was chosen as a putative pathogenic mediator because of its association with obesity, metabolic syndrome and cardiovascular disease. He reviews a series of elegant studies designed to show how CRP signaling through $\mathrm{Fc}$ receptor gamma IIB leads to hypertension, insulin resistance and reduced blood flow to skeletal muscle. The results of these studies have profound implications for the treatment of the metabolic syndrome and atherosclerotic disease in the setting chronic inflammatory diseases such as diabetes and autoimmune disease, where CRP and autoantibody signaling through the Fc receptors is elevated.

Our second speaker is Don Rockey. Dr. Rockey received his Bachelor of Science from the Virginia Polytechnic Institute \& State University and his Medical Degree from the Medical College of Virginia School of Medicine. He completed his residency in medicine and his research and clinical fellowships in gastroenterology at the University of California in San Francisco. He was recruited to Duke University, where he rose to the rank of full Professor and served as Chief of Hepatology and Director of the Duke Liver Center. He was recruited to the University of Texas Southwestern, where he served as Chief of the Division of Digestive and Liver Diseases. He was recruited to the Medical University of South Carolina, where he serves as the Chair of the Department of Medicine. His research is focused on mechanisms of liver fibrosis and portal hypertension and has more recently begun to focus on the role of NO in these processes. His talk ${ }^{4}$ will take a cell and molecular approach to portal hypertension and will elucidate signaling mechanisms that lead to stellate cell contraction in the liver. He begins by reviewing how endothelin production in the cirrhotic liver shifts from endothelial cells to stellate cells, leading to a stellate cell contractile phenotype. He then shifts emphasis to studies in which his laboratory first described the expression of eNOS in sinusoidal endothelial cells and mechanisms of control of eNOS function. Production of NO by eNOS is critical because it counteracts contraction induced by endothelin. He describes work in his laboratory demonstrating the role of and mechanisms for regulation of eNOS in sinusoidal endothelial cells. This work is remarkable because it not only offers mechanisms through which portal hypertension might be targeted in liver disease to treat or prevent portal hypertension but also because the same pathways are likely to be present in the peripheral vasculature.

\section{REFERENCES}

1. Demystified Stuart-Smith K. Nitric oxide. Mol Pathol 2002;55: 360-6. [PubMed: 12456772]

2. O'Meara YM, Brady HR. Lipoxins, leukocyte recruitment and the resolution phase of acute glomerulonephritis. Kidney Int Suppl 1997; 58:S56-61. [PubMed: 9067945]

3. Shaul PW. Role of the endothelium in the metabolic syndrome: IIB or not IIB. Am J Med Sci 2015;349:3-5. [PubMed: 25504220]

4. Rockey DC. Endothelial dysfunction in advanced liver disease. Am J Med Sci 2015;349:6-16. [PubMed: 25559279] 